\title{
Extraction of the Retinal Blood Vessels and Detection of the Bifurcation Points
}

\author{
Manjiri B. Patwari \\ Institute Of \\ Management Studies \\ \& Vivekanand \\ Information \\ technology college \\ campus, \\ Aurangabad (MS), \\ India
}

\author{
Ramesh R. \\ Manza, Ph.d \\ Department of CS \\ and IT \\ B. A.M,Ph.d \\ University, \\ Aurangabad (MS), \\ India
}

\author{
Yogesh M. \\ Rajput \\ Department of CS \\ and IT \\ B. A.M,Ph.d \\ University, \\ Aurangabad (MS), \\ India
}

\author{
Neha K. \\ Deshpande, \\ Ph.d \\ "Director", \\ Guruprasad \\ Netra
}

Rugnalaya pvt. Itd, Aurangabad MS (India),

\author{
Manoj \\ Saswade, \\ Ph.d \\ "Director", \\ Saswade Eye \\ Clinic"
}

Aurangabad(MS

) India (India),

\begin{abstract}
The changes in retinal blood vessels Structure and progression of diseases such as diabetes, hypertension and retinopathy of prematurity (ROP) has been the subject of several large scale clinical studies. Proposed algorithm for the detection and measurement of blood vessels of the retina and finding the bifurcation points of blood vessels is general enough that it can be applied to high resolution fundus photographs. The algorithm proceeds through three main steps 1 . Preprocessing operations on high resolution fundus images 2. For retinal vessel extraction, simple vessel segmentation techniques formulated in the language of 2D Median Filter 3. Minutiae techniques for finding bifurcation points of the extracted blood vessels. Performance of this algorithm is tested using the fundus image database ( 240 images) taken from Dr. Manoj Saswade, Dr.Neha Deshpande and online available databases diaretdb0, diaretdb1 and DRIVE. This algorithm achieves accuracy of $96 \%$ with 0.92 sensitivity and 0 specificity for Saswade database, for diaretdb0 accuracy 95\% with 0.95 sensitivity and 0 specificity, for diaretdb1 accuracy $96 \%$ with 0.96 sensitivity and 0 specificity, and for DRIVE database $98 \%$ accuracy with 0.98 sensitivity and 0 specificity.
\end{abstract}

\section{Keywords}

Blood Vessels, Bifurcation Points 2D Median Filter.

\section{INTRODUCTION}

Proposed algorithm shows the blood vessels extraction and detection of the bifurcation points of the vessels. In this algorithm Image Processing techniques are used for extraction of the retinal blood vessels. Firstly preprocessing operation is performed on high resolution fundus images. Then 2D median filter is used for highlighting the blood vessel. For extraction of the blood vessels thresholding is used. for finding the bifurcation points of the blood vessels Minutiae techniques are performed. For observing the result images are taken from Dr. Manoj Saswade(240 images) and data base is formed, also images from online databases diaretdb0, diaretdb1 and Drive are used.

\section{METHODOLOGY}

Computer assisted diagnosis for various diseases are very common now a days and medical imaging is playing a vital role in such diagnosis. The proposed algorithm has 3 stages, shown in the figure 1 . In first stage preprocessing is done. Blood vessels are highlighted and extracted in the second stage and in the third stage using thresholding technique and Skeletonazation bifurcation points are detected.

\subsection{Preprocessing :}

In this algorithm for enhancement of retinal blood vessels some Image Processing techniques are used. The Preprocessing is done to remove noise from the background and to enhance the image. In the first stage of preprocessing green channel is taken out, because green channel shows high intensity as compare to red and blue. Mathematical formula for finding green channel is shown in the equation 1. Green channel is taken out from RGB image because Green channel has high intensity as compared to Red and Blue. Fundus image and its equivalent green channel image are shown in the figure 2 .

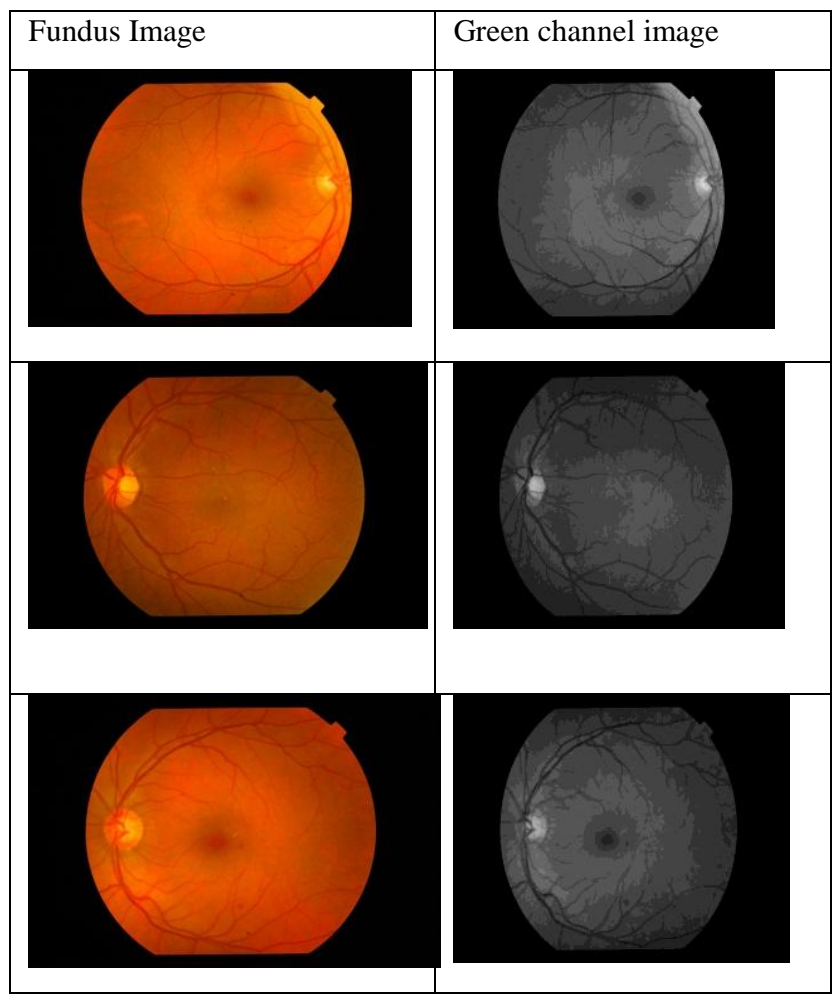

Figure 2: Fundus image and green channel image 


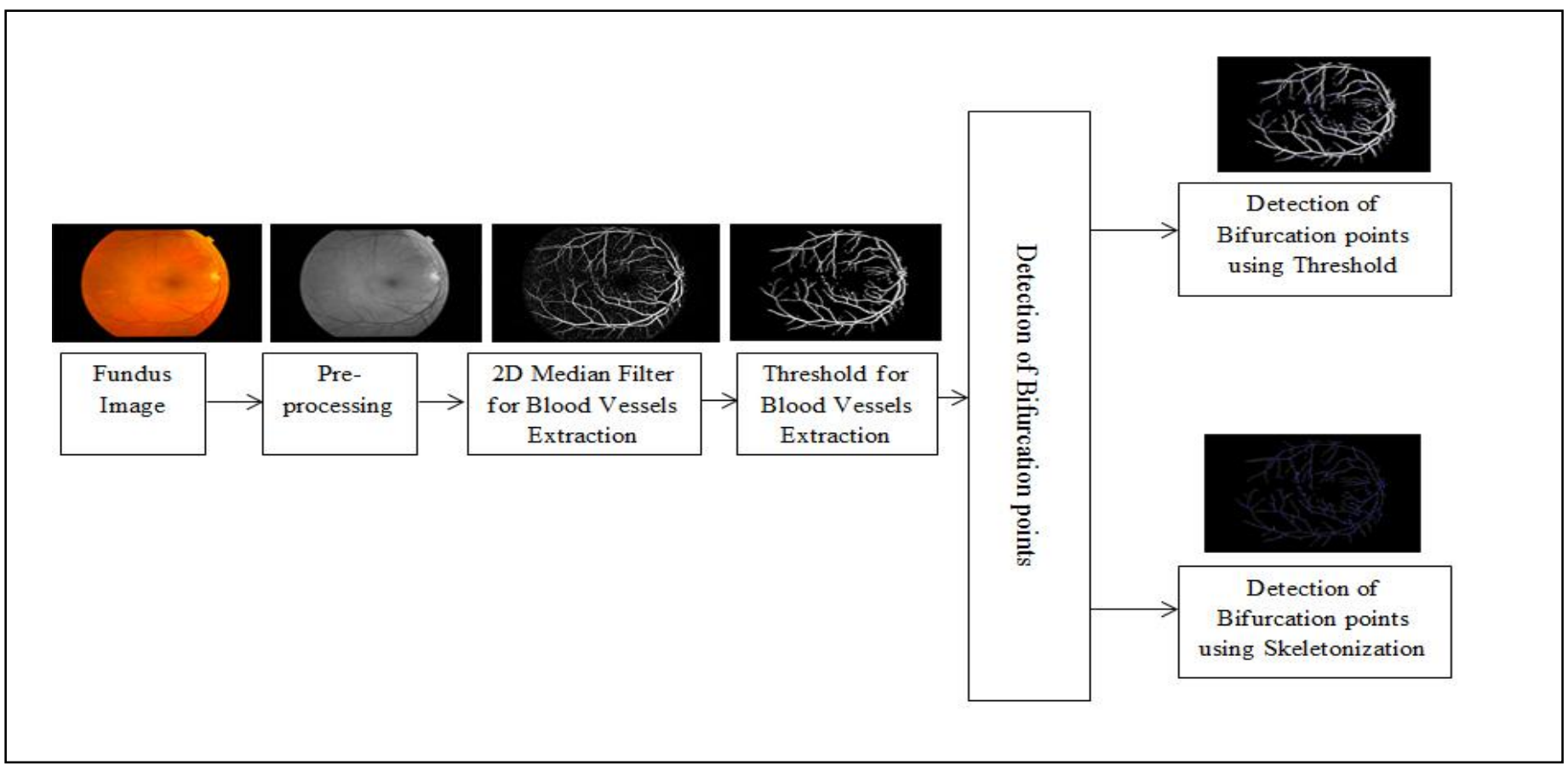

Figure 1: Flow chart for proposed algorithm of Extraction of the Retinal Blood Vessels and Detection of the Bifurcation Points

$$
g=\frac{G}{(R+G+B)}
$$

Here $\mathrm{g}$ is a Green channel and $\mathrm{R}, \mathrm{G}$ and $\mathrm{B}$ are Red, Green and Blue respectively.

\subsection{Blood Vessels}

\subsubsection{Blood Vessels Enhancement}

Then the complement function is used for enhancing the blood vessels of the retina.

$\mathrm{A}^{\mathrm{c}}=\{\omega \mid \omega \notin \mathrm{A}\}$

Here $A^{c}$ is a complement, $\omega$ is the element of $A, \notin$ stands for not an element of $\mathrm{A}$ and $\mathrm{A}$ is set.

Then Histogram equalization function is used for enhancing the complementary image.

$$
\mathrm{h}(\mathrm{v})=\operatorname{round}\left(\frac{\mathrm{cdf}(\mathrm{v})-\mathrm{cdf}_{\min }}{(\mathrm{M} \times \mathrm{N})-\mathrm{cdf}_{\min }} \times(\mathrm{L}-1)\right)
$$

Here $\mathrm{cdf}_{\min }$ is the minimum value of the cumulative distribution function, $\mathrm{M} \times \mathrm{N}$ gives the image's number of pixels and $\mathrm{L}$ is the number of grey levels.

As shown in the figure 3, the Morphological structuring element is used for highlighting the blood vessels of the retina.

$$
I_{\text {dilated }}(i, j)=\max _{f(n, m)=\text { true }} I(i+n, j+m)
$$

$$
I_{\text {eroded }}(i, j)=\min _{f(n, m)=t r u e} I(i+n, j+m) \quad(5)
$$

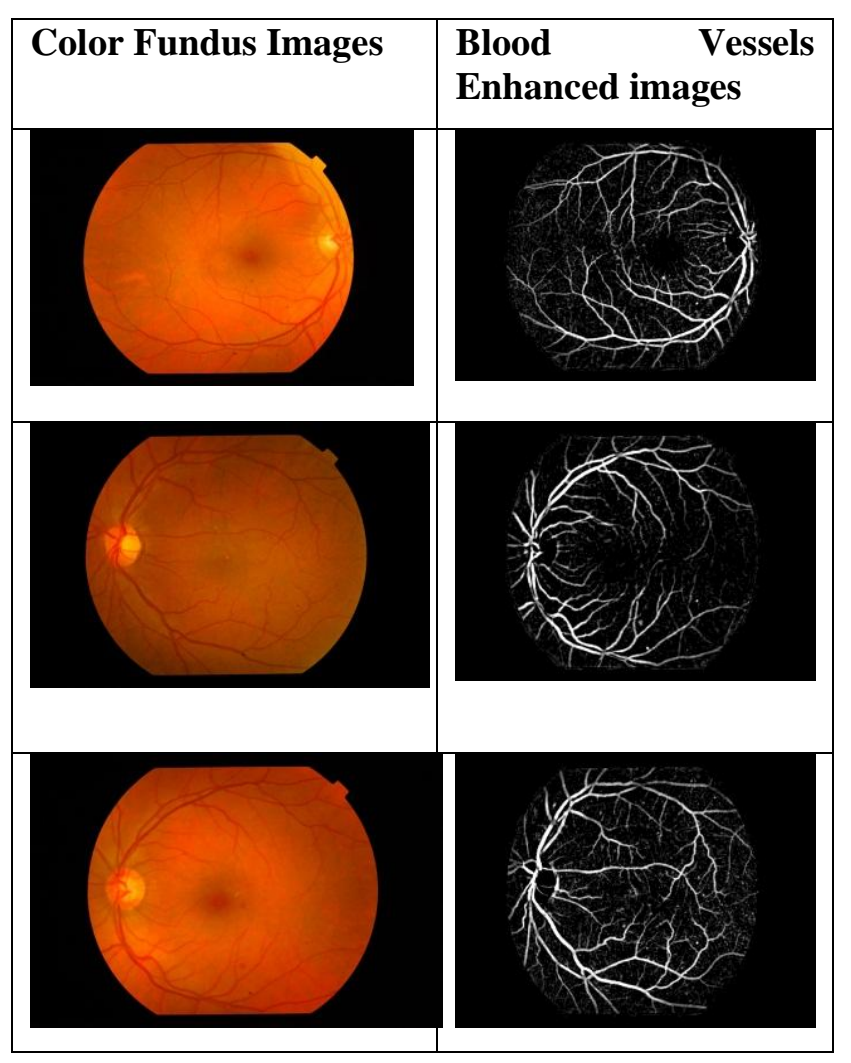

Figure 3: Fundus image and Blood vessels Enhanced images 
The Morphological open function is used for thickening the retinal blood vessels.

$$
\mathrm{A} \circ \mathrm{B}=(\mathrm{A} \ominus \mathrm{B}) \oplus \mathrm{B}
$$

Here $\mathrm{A} \circ \mathrm{B}$ is morphological opening, $\Theta$ is Erosion and $\bigoplus$ is Dilation.

After Dilation 2D median filter is used for highlighting and removing noise from the Morphological open function.

$y[m, n]=\operatorname{median}\{x[i, j],(i, j) \in \omega\}$

Here $\omega$ Represents a neighborhood centered around location $(m, n)$ in the image.

Threshold function is used for extracting the retinal blood vessels, result images are shown in the figure 4 .

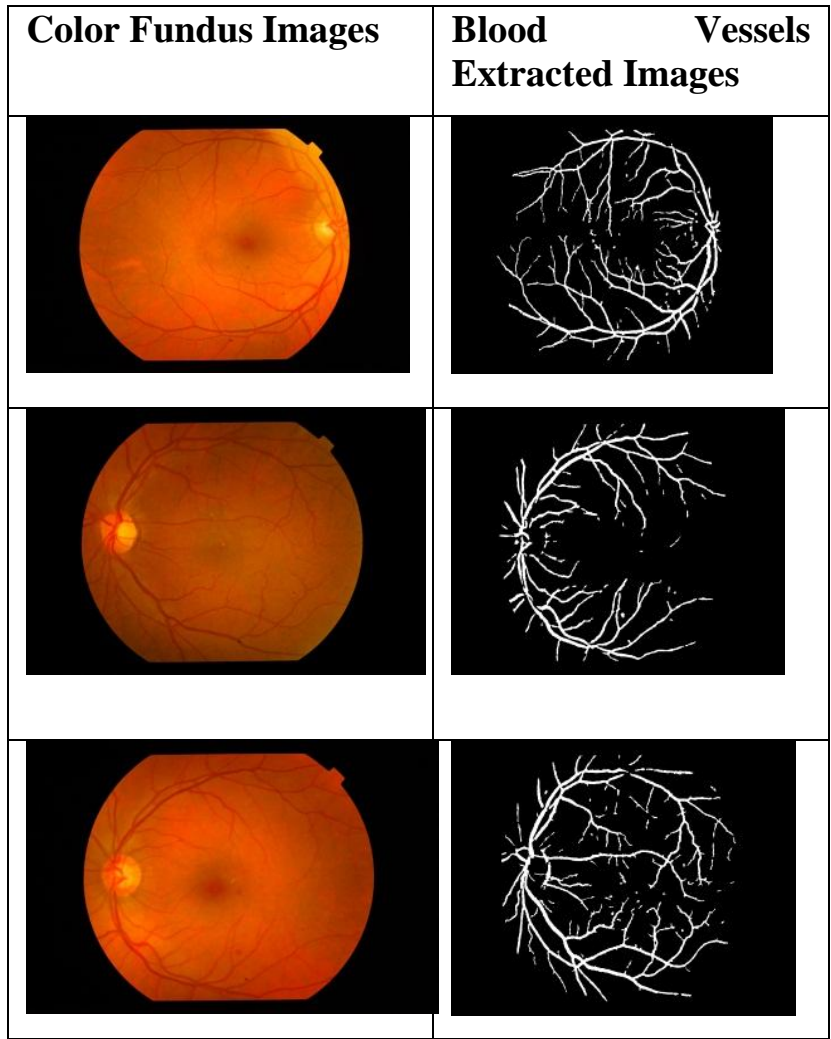

Figure 4: Fundus images and Images obtained using Threshold to Extract Blood Vessels

\subsection{Detection of Bifurcation Point}

\subsubsection{Using Threshold}

Bifurcation points are detected by applying threshold, images are shown in the figure 5 .

$$
\mathrm{T}=\frac{1}{2}(\mathrm{~m} 1+\mathrm{m} 2)
$$

Here $\mathrm{m} 1 \& \mathrm{~m} 2$ are the Intensity Values.

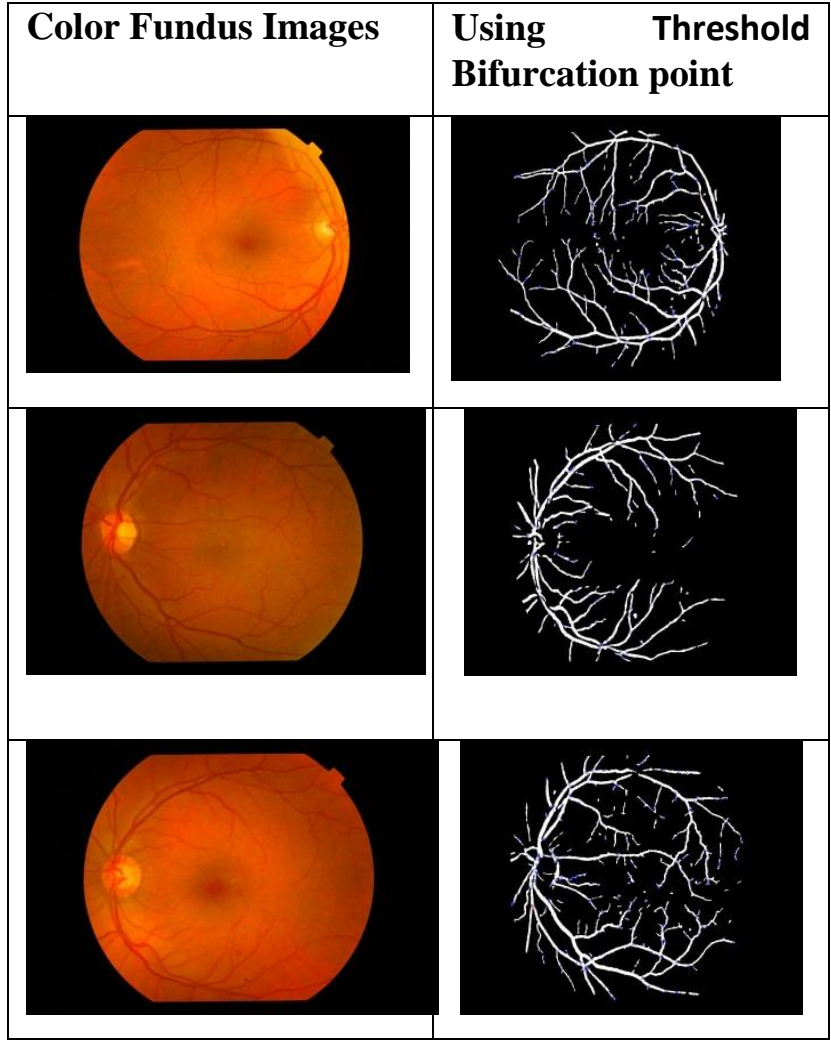

Figure 5: Fundus images and Images showing bifurcation points obtained using Threshold

\subsubsection{Using Skeletonization}

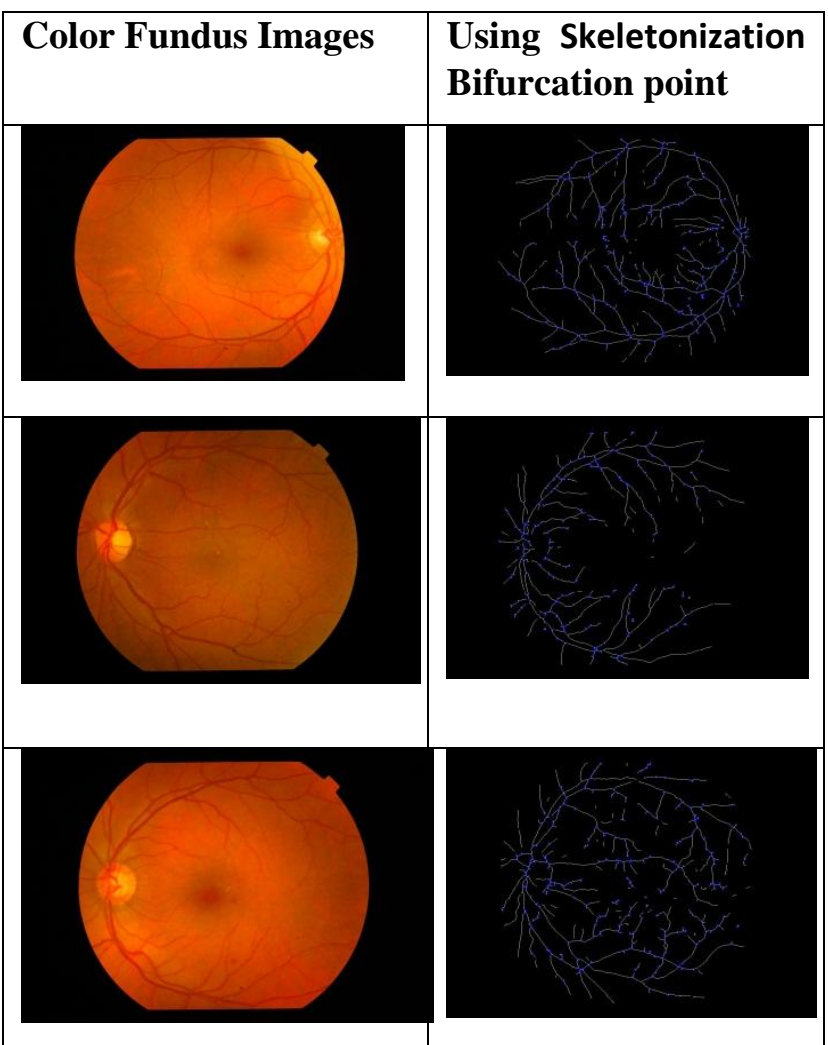

Figure 6: Fundus images and Images showing bifurcation points obtained using Skeletonization 


$$
S(X)=\bigcup_{P>0} \bigcap_{\mu>0}[(X \ominus \rho B)-(X \ominus \rho B) \circ \mu \bar{B}](9)
$$

Result images obtained after Skeletonization are shown in figure 6 .

\section{RESULT}

For this algorithm GUI is designed in MATLAB, as shown in the figure 7, for result analysis Receiver Operating Characteristic Curve (ROC) is used. ROC curve for Saswade database is shown in figure 8 , this algorithm achieves a true positive rate of $96 \%$, false positive rate of $0 \%$ and accuracy score 0.9202. Roc for Diaretdb0 this algorithm achieves a true positive rate of $95 \%$, false positive rate of $0 \%$, and accuracy score of 0.9514 as shown in figure 9 , on diaretdb1 this algorithm achieves a true positive rate of $96 \%$, false positive rate of $0 \%$, and accuracy score of 0.9665 as shown in figure 10 and on DRIVE this algorithm achieves a true positive rate of $98 \%$, false positive rate of $0 \%$, and accuracy score of 0.9802 as shown in figure 11. Table 1 shows Performance Evaluation and table 2 shows accuracy.

Table 1: Performance Evaluation

\begin{tabular}{|l|l|l|}
\hline Test Result & Present & Absent \\
\hline Positive & True Positive (TP) & False Positive (FP) \\
\hline Negative & True Negative (TN) & False Negative (FN) \\
\hline
\end{tabular}

Sensitivity $=\frac{\mathrm{TP}}{\mathrm{TP}+\mathrm{FN}}$

$$
\text { Specificity }=\frac{\mathrm{TN}}{\mathrm{TN}+\mathrm{FN}}
$$

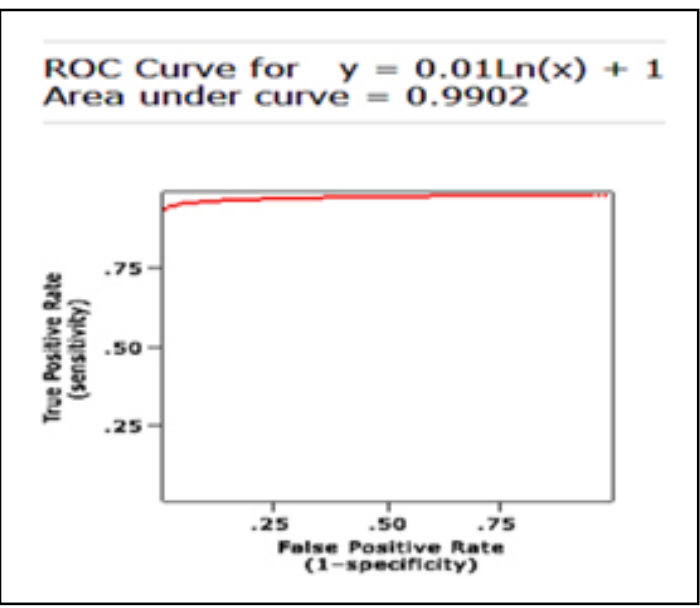

Figure 8: Receiver Operating Characteristics Curve for blood vessel extraction and detecting bifurcation points of vessels on Dr. Saswade's Image Database.
Table 2: Shows Sensitivity, Specificity and Accuracy

\begin{tabular}{|l|l|l|l|l|l|}
\hline $\begin{array}{l}\text { N } \\
\text { o. }\end{array}$ & Database & $\begin{array}{l}\text { Sensit } \\
\text { ivity }\end{array}$ & $\begin{array}{l}\text { Specif } \\
\text { icity }\end{array}$ & ROC & Accuracy \\
\hline 1 & Saswade & 0.92 & 0 & 0.9202 & $96 \%$ \\
\hline 2 & Diaretdb0 & 0.95 & 0 & 0.9514 & $95 \%$ \\
\hline 3 & Diaretdb1 & 0.96 & 0 & 0.9665 & $96 \%$ \\
\hline 4 & DRIVE & 0.98 & 0 & 0.9802 & $98 \%$ \\
\hline
\end{tabular}

ROC Curve for $y=0.01 \ln (x)+1$
Area under curve $=0.9514$

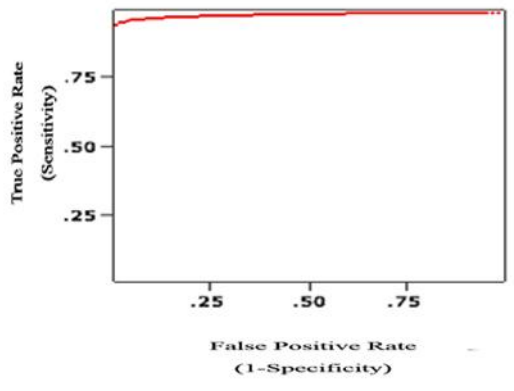

Figure 9: Receiver Operating Characteristics Curve for blood vessel extraction and detecting bifurcation points of vessels on online available Database diaretdb0

ROC Curve for $y=0.01 \operatorname{Ln}(x)+1$

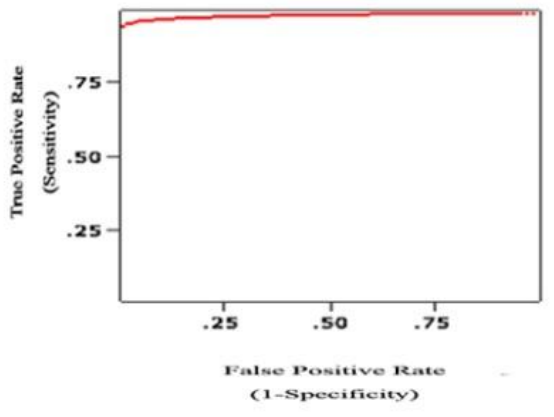

Figure 10: Receiver Operating Characteristics Curve for blood vessel extraction and detecting bifurcation points of vessels on online available Database diaretdb1 
ROC Curve for $y=0.01 \operatorname{Ln}(x)+1$

Area under curve $=0.9802$

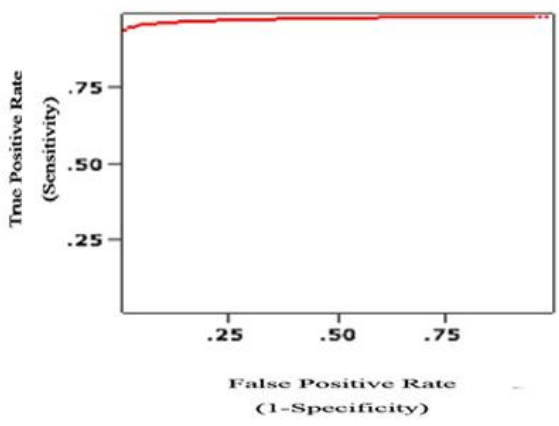

Figure 11: Receiver Operating Characteristics Curve for blood vessel extraction and detecting bifurcation points of vessels on online available Database diaretdb1

\section{DISCUSSION}

For this algorithm Image processing techniques are used. Initially Green channel from RGB image is taken out because Green channel has high intensity as compare to Red and Blue. On the green channel different operations are performed using functions like, Complement function to highlight the Green channeled image, Histogram equalization for enhancement of the complemented image, Morphological structuring element, Morphological opening for thickening the retinal blood vessels, 2D Median filter for removing noise, for extracting the retinal blood vessels Threshold function and for finding the bifurcation points of the blood vessels the minutiae techniques. For manipulating these techniques MATLAB 2012a is used and with the help of this tool one GUI is designed for retinal blood vessel the retinal blood vessels extraction and bifurcation point detection.

\section{CONCLUSION}

In this algorithm Image processing techniques are used for extracting the blood vessels of the retina and detecting the bifurcation points of the extracted blood vessels. For performing these techniques database from Dr. Manoj Saswade, Dr. Neha Deshpande and online available databases diaretdb0, diaretdb1 and DRIVE are used. This algorithm for Saswade database achieves accuracy of $96 \%$ with 0.92 sensitivity and 0 specificity, for diaretdb0 accuracy 95\% sensitivity and specificity 0, accuracy $96 \%$ with 0.96 sensitivity and specificity 0 for diaretdb1, and for DRIVE $98 \%$ with 0.98 sensitivity and specificity 0 respectively.

\section{ACKNOWLEDGMENTS}

We are thankful to University Grant Commission (UGC) for providing us a financial support for the Major Research Project entitled "Development of Color Image Segmentation and Filtering Techniques for Early Detection of Diabetic Retinopathy" F. No.: $41-651 / 2012$ (SR). We are thankful to "Saswade Eye Clinic" Aurangabad and "Guruprasad Netra Rungnalaya pvt. Ltd", Samarth Nagar, Aurangabad for proving the Database and accessing the Result.

\section{REFERENCES}

[1] Knudtson MD, Klein BEK, Klein R, Wong TY, Hubbard $\mathrm{LD}$, et al. Variation associated with measurement of retinal vessel diameters at different points in the pulse cycle. Br J Ophthalmol.2004;88:57-61.

[2] Wong LY, U'Rajendra A, Venkatesh YV, Caroline C, Lim CM, Ng EYK: Identification of different stages of Diabetic retinopathy using retinal optical images. Inf Sci 2008, 178:106-121.

[3] Rangaraj M. Rangayyan,1 Xiaolu Zhu,1 Fábio J. Ayres,1 and Anna L. Ells2 "Detection of the Optic Nerve Head in Fundus Images of the Retina with Gabor Filters and Phase Portrait Analysis" Journal of Digital Imaging, Vol 23, No 4 (August), 2010: pp 438 Y453

[4] S. Jiméneza,P. Alemanya, I. Fondónb, A. Foncubiertab, B. Achab and C. Serranob "Automatic detection of vessels in color fundus images" (C) 2009 Sociedad Española de Oftalmología. Published by Elsevier España, s.larchsocespoftalmol. 2010;85(3):103-109

[5] Ana MM, Aurelio C: Segmentation of Retinal Blood Vessels by Combining the Detection of Centerlines And Morphological Reconstruction. IEEE Trans Medical imaging 2006, 25:1200-1213.

[6] Fischer JG, Mewes H, Hopp HH, Schubert R. Analysis of pressurized resistance vessel diameter changes with a low cost digital image processing device. Comput Meth Prog Bio. 1996;50:23-30.

[7] Patton N, Aslam T, Macgillivray T, Pattie A, Deary IJ, et al. Retinal vascular image analysis as a potential screening tool for cerebrovascular disease: a rationale based on homology between cerebral and retinal microvasculatures. J Anat. 2005;206:319-348.

[8] R. Winder, P. Morrow, I. McRitchie, J. Bailie, and P. Hart, "Algorithms for digital image processing in diabetic retinopathy," Computerized Med. Imag. Graph., vol. 33, pp. 608-622, 2009.

[9] H. Li, W. Hsu, M. L. Lee, and H. Wang, "A piecewise gaussian model for profiling and differentiating retinal vessels," International Conference on Image Processing, 2003.

[10] Manjiri B. Patwari, Dr. Ramesh R. Manza, Dr. Manoj Saswade and Dr. Neha Deshpande, "A Critical Review of Expert Systems for Detection and Diagnosis of Diabetic Retinopathy", Ciit International Journal of Fuzzy Systems, February 2012, DOI: FS022012001 ISSN 0974-9721, 0974-9608. (IF 0.441).

[11] Yogesh M. Rajput, Ramesh R. Manza, Manjiri B. Patwari, Neha Deshpande, "Retinal Blood Vessels Extraction Using 2D Median Filter", Third National Conference on Advances in Computing(NCAC2013), 5th to 6th March 2013, School of Computer Sciences, North Maharashtra University, Jalgaon-425001 (MS) India.

[12] Yogesh M. Rajput, Ramesh R. Manza, Manjiri B. Patwari, Neha Deshpande, "Retinal Optic Disc Detection Using Speed Up Robust Features", National Conference on Computer \& Management Science [CMS-13], April 25-26, 2013, Radhai Mahavidyalaya, Auarngabad431003(MS India). 
[13] A. Hoover, V. Kouznetsova and M. Goldbaum, Locating Blood Vessels in Retinal Images by Piece-wise Threhsold Probing of a Matched Filter Response", IEEE Transactions on Medical Imaging, vol. 19 no. 3, pp. 203210, March 2000.

[14] A. Hoover and M. Goldbaum, "Locating the optic nerve in a retinal image using the fuzzy convergence of the blood vessels", IEEE Transactions on Medical Imaging, vol. 22 no. 8, pp. 951-958, August 2003.

[15] A. Hoover and M. GoldBaum. Locating the optic nerve in a retinal image using the fuzzy convergence of the blood vessels. IEEE Trans. on Medical Imaging

[16] M. Sofka and C. V. Stewart. Retinal vessel extraction using multiscale matched filters, confidence and edge measures. IEEE Trans. on Medical Imaging, 25(12):1531-1546, 2006. 2

[17] J. Staal,M. D. Abramoff,M. Niemeijer,M. A. Viergever, and B. V. Ginneken. Ridge-based vessel segmentation in color images of the retina. IEEE Trans. on Medical Imaging, 23(4):501- 509, 2004.

[18] J. L. Company, "Grading diabetic retinopathy from stereoscopic color fundus photographs - an extension of the modified airlie house classification," ETDRS Report No. 10, Ophthalmology, the Journal of the American Academy of Ophthalmology, vol. 98, no. 5, p. 78, May 1991.

[19]O.Chutatape, L.Zheng, and S.M.Krishnan, "Retinal blood vessel detection and tracking by matched gaussian and kalman filters," Proceedings of the 20th Annual International Conference of the IEEE Engineering in Medicine and Biology society, vol. 20, no. 6, pp. 3144 3149, 1998

[20] H. Li, W. Hsu, M. L. Lee, and H. Wang, "A piecewise gaussian model for profiling and differentiating retinal vessels," International Conference on Image Processing, 2003.

[21] M. Turner, "Texture discrimination by gabor functions," Biological Cybernetics, vol. 55, pp. 71-82, 1986

[22] A.Bovik, M. Clark, and W. Geisler, "Multichannel texture analysis using localized spatial filters," IEEE Trans. PAMI, vol. 12, no. 1, pp. 55-73, 1990.

[23] A.Jain and F. Farrokhnia, "Unsupervised texture segmentation using gabor filters," Intl. Journal of Computer Vision, pp. 279-302, 1988.

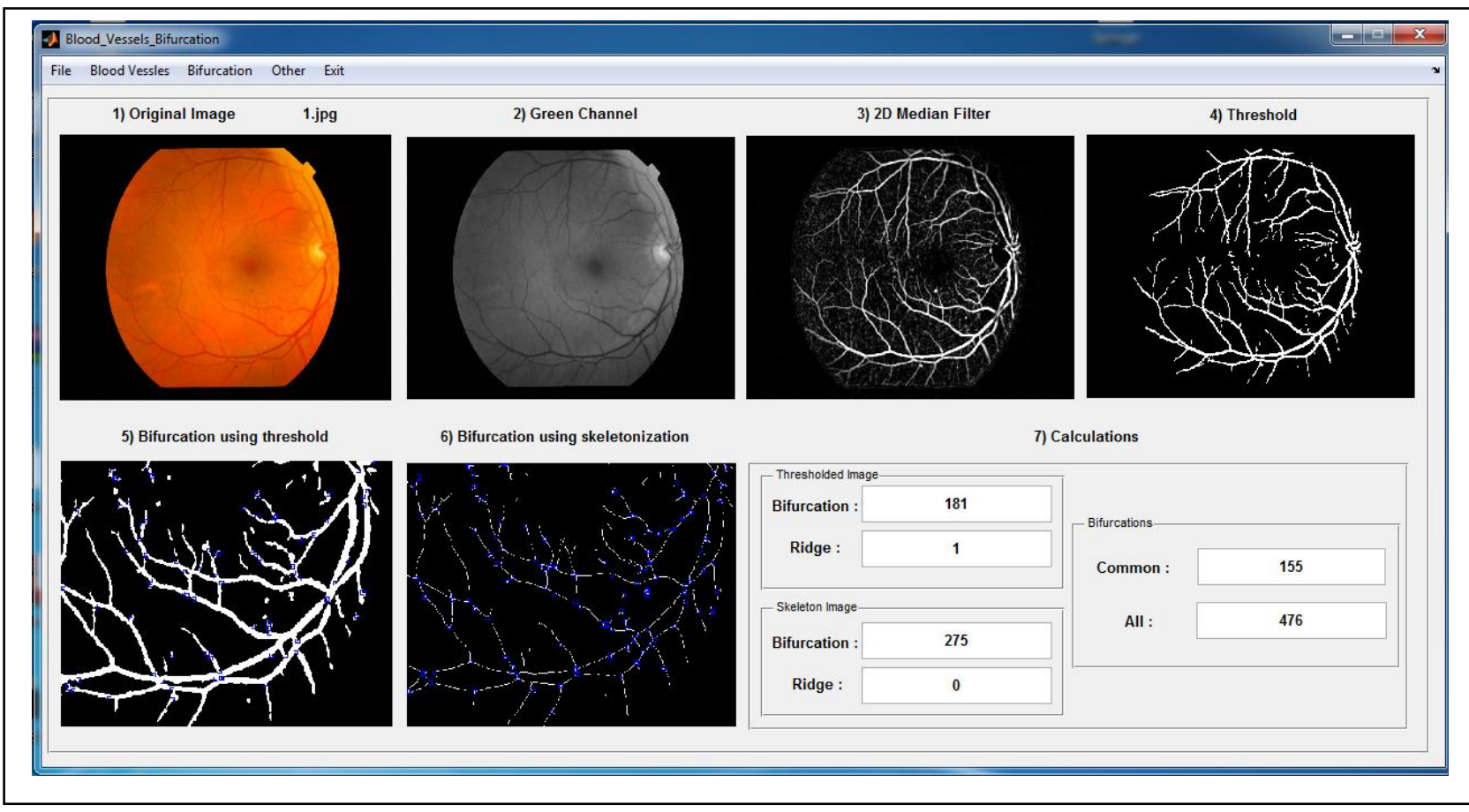

Figure 7: GUI for Blood Vessels Extraction and Detection of Bifurcation Point 